\title{
Omics in marine biotechnology
}

Marine biotechnology emerged in the 1980s, and since then it has been gradually gaining momentum to meet growing demands that cannot be satisfied by terrestrial sources alone. Marine biotechnology has been widely applied to tap the unique biodiversity found in oceans and corresponding resources. Although marine biotechnology is relatively young, it has made considerable contributions to sustainable development on social, economic and environmental fronts. In the mid-1990s, the 'omics' revolution triggered major changes in biological research and its application to biotechnology. Omics aims to combine all the information acquired from different hierarchies that control vital processes to investigate the nature of numerous biological phenomena at the molecular level.

This special topic focuses on several omics in research to investigate basic biological systems in marine biotechnology. Special attention is devoted to genomics, transcriptomics, proteomics, metabolomics and nutrigenomics. It is hoped that this new information will contribute to increased success in marine science and related industries. This topic contains 12 papers produced by research groups from China, Germany, and Korea. To help readers, we have arranged the papers according to their research topics: genomics [1-3]; transcriptomics [4]; proteomics [5-9]; metabolomics [10,11], and nutrigenomics [12].

The objective of this topic is to offer a platform for marine biological scientists to present recent advances in the discovery and development of omics in marine biotechnology, providing a large-scale, holistic approach to understanding life in encapsulated "omes". In this special topic, several genomes, including major histocompatibility complex genes [2], phytochrome genes [3] and immune- and antiviral-related genes [4], have been systematically investigated based on functional genomic studies, providing targets for further structural and functional analyses. Wang and co-workers [7] present a rapid and simple protein labeling approach, which could be a tool for further proteomic studies. Zhang and Chen [1] offer us a comprehensive overview of metabolomics, from concept, approaches and widespread application to marine biology. In addition to the three subdisciplines of omics mentioned above, this topic also focuses on nutrigenomics in marine biotechnology [12]. This defines the causality/relationship between specific nutrients and nutrient regimens of marine food by determining the mechanisms involved in the effects of nutrients or the effects of a nutritional regimen.

We would like to sincerely thank the authors for their excellent contributions. Their work has been crucial in highlighting the diverse and exciting opportunities in this field of research, and provides a decisive contribution for the further development of marine biotechnology. We also extend our appreciation to Mr. PU Yang and Dr. JIANG Tinging, who generously provided extremely valuable assistance during the review process.

1 Zhang X, Chen H. Metabolomics: Concept, methods and potential prospect in marine biology. Chin Sci Bull, 2012, 57: 3253-3261

2 Du M, Chen S L, Liu Y H, et al. MHC polymorphism and disease resistance to Edwardsiella tarda in six turbot (Scophthalmus maximus) families. Chin Sci Bull, 2012, 57: 3262-3269

3 Shi F, Li N, Liu S F, et al. Sequence analysis of the Microcystis aeruginosa FACHB-912 phytochrome gene supports positive selection in Cyanobacteria. Chin Sci Bull, 2012, 57: 3270-3276

4 Yan Y, Cui H C, Wei J G, et al. Functional genomic studies on an immune and antiviral related gene of MyD88 in orange-spotted grouper, Epinephelus coioides. Chin Sci Bull, 2012, 57: 3277-3287

5 Wu Y Z, Kim W, Kim S W, et al. Expression and self-assembly of Herterocapsa circularisquama RNA virus-like particle symthesized in Pichia pastoris. Chin Sci Bull, 2012, 57: 3288-3293

6 Guan X Y, Zhang W J, Chi X Y, et al. Combinational biosynthesis and characterization of a fluorescent $82 \beta$-phycocyanin of Spirulina platensis. Chin Sci Bull, 2012, 57: 3294-3299

7 Wang X H, Fan X T, Schröder H C, et al. Flashing light in sponges through their siliceous fiber network: A new strategy of “neuronal transmission” in animals. Chin Sci Bull, 2012, 57: 3300-3311

8 Zhang L L, Li L, Zhang G F. Sequence variability of fibrinogen-related proteins (FREPs) in Crassostrea gigas. Chin Sci Bull, 2012, 57: 3312-3319

9 Li C, Wang D Z, Dong H P, et al. Proteomics of a toxic dinoflagellate Alexandrium catenella DH01: Detection and idenfication of cell surface proteins using fluorescent labeling. Chin Sci Bull, 2012, 57: 3320-3327

10 Wang D Z, Li L, Wang M H, et al. Proteomic analysis of toxic dinoflagellate Alexandrium catenella under different growth phases and conditions. Chin Sci Bull, 2012, 57: 3328-3341 
11 Ouyang L L, Du D H, Yu S Y, et al. Expressed sequence tags analysis revealing the taxonomic position and fatty acid biosynthesis in an oleaginous green microalga, Myrmecia incise Reisigl (Trebouxiophyceae, Chlorophyta). Chin Sci Bull, 2012, 57: 3342-3352

12 Ye Y F, Zhang L M, Tang H R, et al. Survey of nutrients and quality assessment of crab paste by ${ }^{1} \mathrm{H}$ NMR spectroscopy and multivariate data analysis. Chin Sci Bull, 2012, 57: 3353-3362

QIN Song

Yantai Institute of Coastal Zone Research, Chinese Academy of Sciences, Yantai 264003, China

WATABE Shugo

Department of Aquatic Bioscience, Graduate School of Agricultural and Life Sciences, The University of Tokyo, Bunkyo-ku, Tokyo 113-8657, Japan Kitasato University School of Marine Biosciences, Minami-ku, Sagamihara, Kanagawa 252-0373, Japan

LIN HanZhi

Key Laboratory of Experimental Marine Biology, Institute of Oceanology, Chinese Academy of Sciences, Qingdao 266071, China

Open Access This article is distributed under the terms of the Creative Commons Attribution License which permits any use, distribution, and reproduction in any medium, provided the original author(s) and source are credited. 